\title{
The gas permeability coefficient of the cyanobacterial gas vesicle wall
}

\author{
A. E. Walsby, ${ }^{1 *}$ N. P. ReVsbeCh ${ }^{2}$ and D. H. GRIFFeL ${ }^{3}$ \\ ${ }^{1}$ Department of Botany, University of Bristol, Woodland Road, Bristol BS8 IUG, UK \\ ${ }^{2}$ Department of Ecology and Genetics, University of Aarhus, Ny Munkegade, DK-8000 Aarhus C, Denmark \\ ${ }^{3}$ Department of Mathematics, University of Bristol, University Walk, Bristol BS8 ITW, UK
}

(Received 15 August 1991; revised 27 November 1991; accepted 5 December 1991)

\begin{abstract}
The gas permeability coefficient of the cyanobacterial gas vesicle wall has been determined by comparing the concentration gradient of oxygen gas in a film of gas vesicles with the gradient in an underlying film of agar supported over an oxygen atmosphere. The gradients were determined with an oxygen microelectrode. The value of the gradient in aqueous agar was 0.81 of that in a suspension in which gas vesicles occupied 0.35 of the total volume. From this it was calculated that the notional diffusivity of oxygen through the gas vesicle was equivalent to 0.53 of the diffusivity in water. The permeability coefficient of the gas vesicle membrane is calculated to be $\kappa=32 \mathrm{~mm} \mathrm{~s}^{-1}$, the rate coefficient for filling the gas vesicle by diffusion is $\alpha=2.4 \times 10^{6} \mathrm{~s}^{-1}$ and the folding time for equilibration of gas into a gas vesicle is $t_{\mathrm{e}}=0.4 \mu \mathrm{s}$. The permeability coefficient is about 100-fold higher than the minimum value set by previous pressure rise experiments, and confirms that gas vesicles could not store gas. The measurements also show, however, that randomly oriented gas vesicles would not provide a diffusion channel with a diffusivity higher than that in water, although a layer of gas vesicles oriented with their long axes parallel to the diffusion gradient would provide a diffusivity 3.5-fold higher. The determination of the diffusivity was made with a theory, based on diffusion equations, which can be used in the determination of the diffusivity through other cell organelles.
\end{abstract}

\section{Introduction}

The gas vesicles of cyanobacteria have the form of hollow cylindrical tubes closed at each end by hollow conical caps. The wall of the gas vesicle is impermeable to liquid water but is freely permeable to gas (Walsby, 1969). As a consequence of this, the hollow space in the structure is usually filled with air at atmospheric pressure, in equilibrium with the air dissolved in the surrounding water. The wall structure itself is quite rigid and withstands considerable hydrostatic pressures with little change in the overall volume of the gas vesicle (Walsby, 1982a, 1991). The inner surface of the gas vesicle is hydrophobic and water is prevented from entering the gas space by surface tension. (See Walsby, 1972 , for a review of these properties.)

Gas vesicles are much less dense than water and they provide many planktonic bacteria with buoyancy (Walsby, 1972). There has been speculation that they might also have other, secondary functions. The idea that they might have a role in storing particular gases was dismissed by the finding that they were permeable to

\footnotetext{
* Author for correspondence. Tel. (0272) 303761.
}

gases, but this led to another suggestion, that they might act as gas-conducting channels that would increase the rate at which gases were transported by diffusion (Taylor et al., 1973). This may not have any significance in individual cells because their dimensions are so small (usually $<5 \mu \mathrm{m}$ ) that gases such as oxygen, with a diffusivity (diffusion coefficient) into water $(D)$ of $2000 \mu \mathrm{m}^{2} \mathrm{~s}^{-1}$, will diffuse across them in less than a few milliseconds (Walsby, 1985). (The folding time, $t_{\mathrm{e}}$, for diffusion across a distance $x$ is given by $t_{\mathrm{e}}=x^{2} / 4 D$, so that when $x=5 \mu \mathrm{m}, t_{\mathrm{e}}=3.1 \mathrm{~ms}$.) A higher diffusivity might, however, be significant in promoting gas exchange in colonies, which can measure $1 \mathrm{~mm}$ or more across (when $t_{\mathrm{e}}$ would be $125 \mathrm{~s}$ ). In colonies of the filamentous cyanobacteria Trichodesmium or Aphanizomenon, for example, it is conceivable that the gas vesicles might provide diffusion channels for oxygen or nitrogen along the trichomes, from the centre to the periphery of the colony. Such increased diffusion rates, if they occurred, would be relevant to the suggestion that anaerobic zones in the centre of such colonies permit nitrogen fixation to occur (Carpenter \& Price, 1976).

The original discovery that gas vesicles were permeable to gas was made using modified Warburg respiro- 
meters to measure the amounts of gas exchanged by a gas-vacuolate cell suspension, following a change in pressure of the overlying atmosphere (Walsby, 1969). The results indicated that gas diffused freely in and out of the gas vesicles, but because of the length of diffusion path through thick layers of suspension the rate of gas exchange from the gas vesicles could not be measured. Further evidence of gas permeability was obtained from experiments in which gas pressures were raised rapidly over suspensions of isolated gas vesicles (Walsby, 1971); gas vesicles deep in the suspension instantaneously collapsed when the pressure reached their critical collapse pressure, but those near the surface of the suspension survived intact because, as the gas pressure rose, gas diffused into the suspension and entered the gas vesicles, balancing part of the pressure rise on the outside. In experiments performed over small drops of suspension it was found that the gas could equilibrate through a shallow layer of gas vesicles in less than a second, but here again the water layer offered the main barrier to diffusion.

To avoid this complication, similar experiments were performed with dry films of intact gas vesicles (Walsby, 1984). The dried gas vesicles remained intact even after very rapid gas pressure rises of 50 bar in about $2 \mathrm{~ms}$. From this it was calculated that the minimum rate coefficient for the whole gas vesicle to fill with gas by diffusion must exceed $\alpha=22 \times 10^{3} \mathrm{~s}^{-1}$ and the gas vesicle membrane must have a permeability coefficient exceeding $\kappa=332 \mu \mathrm{m} \mathrm{s}^{-1}$. It was emphasized that these were minimum values and that the actual values might be very much higher. However, this type of experiment cannot be used to investigate much higher values; apart from the technical difficulties of manipulating higher rates of pressure rise, theoretical difficulties may also arise. At high pressures the Boltzmann mean path of the gas molecules may approach the size of pores in the gas vesicle membrane so that gas no longer enters by effusion (diffusion under a pressure gradient) and the permeability values obtained will no longer correspond to the permeability coefficient relevant to gas diffusion at normal pressures.

In discussing the possible role of gas vesicles facilitating gas diffusion across cells, Walsby (1984) calculated that the length $\left(L_{\mathrm{w}}\right)$ of a water layer with the same permeability as a single layer of gas vesicles would be $2 D_{\mathrm{w}} / \kappa$, where $D_{\mathrm{w}}$ is the gas diffusivity through water, $2 \times 10^{3} \mu \mathrm{m}^{2} \mathrm{~s}^{-1}$. ( $L_{\mathrm{w}}$ is proportional to $2 / \kappa$ because the gas must pass the gas vesicle membrane twice.) If $\kappa$ were equal to $322 \mu \mathrm{m} \mathrm{s}^{-1}$, the minimum value, then $L_{\mathrm{w}}$ would be equal to $12 \mu \mathrm{m}$, which is considerably greater than the mean diffusion pathlength across a gas vesicle; such a value for $\kappa$ would not therefore result in shorter diffusion times through gas vesicles than through water.
Walsby (1984) pointed out that by comparing the permeability of a very concentrated gas vesicle suspension with that of water it should be possible to determine the actual permeability coefficient of the gas vesicle. We have now performed such experiments by measuring the concentration gradients $(\mathrm{d} C / \mathrm{d} x)$ of oxygen equilibrated through contiguous layers of gas vesicle suspension and of water immobilized in agar, using the oxygen microsensor techniques described by Revsbech (1989). The theory behind the measurements is as follows.

A thin layer of gas vesicle suspension supported on a layer of agar is placed between a gas phase of pure oxygen below and air above. By diffusion, a concentration gradient of oxygen is established across the two layers. At steady state there is a constant flux, $J$, throughout the two layers, which according to Fick's first law is given by

$$
J=-D_{\mathrm{w}}\left(\mathrm{d} C_{\mathrm{w}} / \mathrm{d} x\right)=-D_{\mathrm{s}}\left(\mathrm{d} C_{\mathrm{s}} / \mathrm{d} x\right)
$$

where $D_{\mathrm{w}}$ and $D_{\mathrm{s}}$ are the diffusivities of oxygen, and $C_{\mathrm{w}}$ and $C_{\mathrm{s}}$ are the oxygen concentrations at depth $x$, in water and the gas vesicle suspension, respectively. (The diffusivity of oxygen in $1 \%$ aqueous agar is virtually identical to that in water; see Revsbech 1989.) Because, at steady state, the flux is constant throughout the two layers, the slopes $\mathrm{d} C / \mathrm{d} x$ for each diffusion gradient will be constant and the ratio of the slopes will be the reciprocal of the ratio of the diffusivities.

We have determined $D_{\mathrm{s}}$, the diffusivity of oxygen in a suspension of gas vesicles isolated from the cyanobacterium Microcystis sp., and have found that it is slightly less than $D_{\mathrm{w}}$, the diffusivity in water. It appears, therefore, that randomly oriented gas vesicles cannot provide a gasconducting channel. We determined $\phi_{v}$, the proportion of the suspension that was occupied by gas vesicles, and from this ratio and the diffusivity in the suspension, we have determined $\kappa$, the permeability coefficient of the gas vesicle membrane.

\section{Methods}

Isolation of gas vesicles. Gas vesicles were isolated from the cyanobacterium Microcystis sp. BC $84 / 1$, by centrifugally accelerated flotation following cell lysis by lysozyme, as described by Walsby (1974) and Hayes (1988). After filtering the purified gas vesicles through Nuclepore membrane filters of pore size $1 \mu \mathrm{m}$, they were further concentrated by repeated centrifugation with the clear subnatant solution removed from under the floating gas vesicles at each step using a fine cannula attached to a $1 \mathrm{ml}$ syringe. Before the final centrifugation the suspension was mixed with 0.1 vol. $50 \mathrm{mM} \mathrm{HgCl}_{2}$, in $50 \mathrm{~mm}$ $\mathrm{KH}_{2} \mathrm{PO}_{4}$ at $\mathrm{pH} 7 \cdot 0$, to act as a preservative. The gas vesicles remained intact in this solution. The final suspension formed a thick 'cream' comprising about $35 \%$ gas vesicles by volume (see below), which although rather viscous could be transferred by Pasteur pipette to the diffusion chamber. 


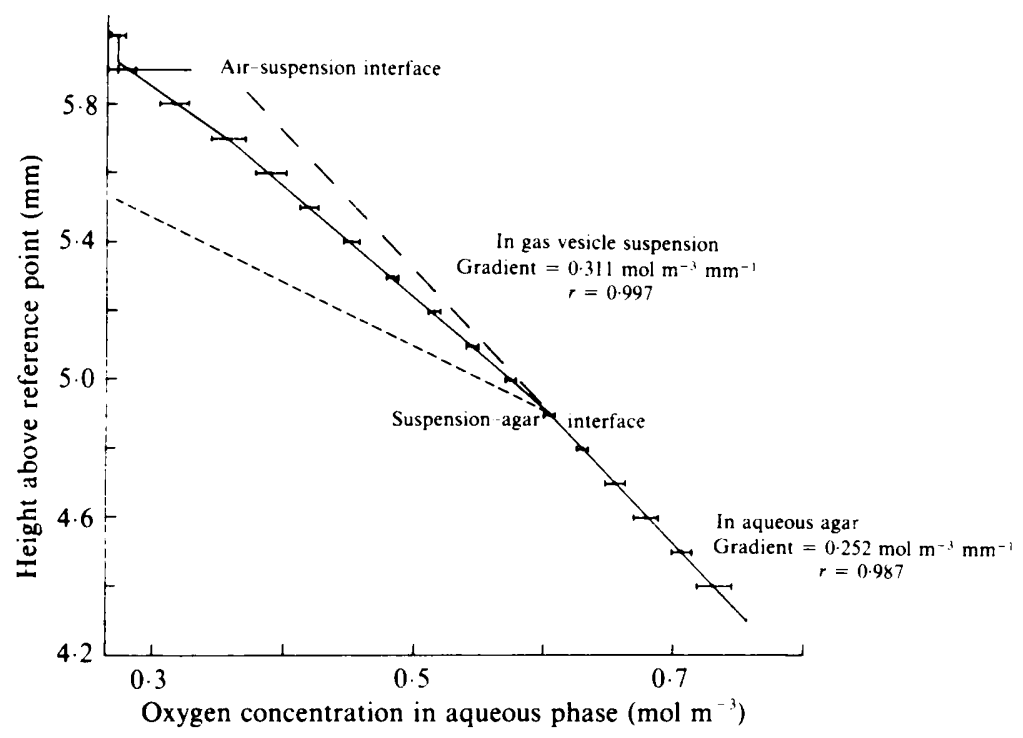

Fig. 1. Oxygen concentration profile from air, through a layer of gas vesicle suspension overlying a layer of aqueous agar suspended above an atmosphere of pure oxygen (means and standard deviations of three measurements). The long-dashed line indicates the extrapolated gradient in agar and the short-dashed line indicates the gradient expected for a suspension of impermeable glass beads with the same volume fraction of water as the gas vesicle suspension. The measurements were made at heights above a reference point on the micromanipulator stand.

\begin{abstract}
Measurement of gas vesicle volume. A sample of the gas vesicle suspension, transferred with a Terumo microsyringe without its detachable cannula, was diluted with $6 \mathrm{~mm}-\mathrm{NH}_{4} \mathrm{HCO}_{3}$. Samples were put in a thermostatted gas vesicle compression tube with a constantbore capillary of nominal internal diameter $200 \mu \mathrm{m}$, and exposed to a pressure of $1.2 \mathrm{MPa}$. The gas vesicle gas volume was determined from the change in position of the meniscus as described by Walsby $(1982 a)$. The diameter of the capillary of the compression tube was subsequently calibrated by filling a measured length with mercury, which was then emptied out and weighed. The radius was $100.43 \pm 0.57 \mu \mathrm{m}(95 \%$ confidence limits).
\end{abstract}

Determination of oxygen concentration gradients. The relative diffusivity of oxygen through the gas vesicle suspension was determined with the use of the diffusion chamber described in Fig. 1 of Revsbech (1989). A layer of $1 \%(w / v)$ agar about $1 \mathrm{~mm}$ deep was deposited on a $2.0 \mathrm{~cm}$ diameter silicone rubber membrane supported on a $0.2 \mathrm{~mm}$ mesh brass screen. A $1 \mathrm{~mm}$ layer of the gas vesicle suspension was then placed over the agar. An atmosphere of pure oxygen gas was introduced in the tube below the mesh screen and the chamber was placed overnight in a 500 $\mathrm{ml}$ beaker with a water-saturated atmosphere, closed by Saran wrap, to allow a stable oxygen diffusion gradient to establish across the agar and gas vesicle layers.

The following morning an oxygen microsensor with a $7 \mu \mathrm{m}$ tip (Revsbech \& Ward, 1983) was lowered by a micromanipulator through a hole in the Saran wrap covering and was advanced in $0.1 \mathrm{~mm}$ steps down through the gas vesicle suspension and agar layers, measuring the oxygen concentration at each step. Three such profiles were measured at different points over the gas vesicle surface.

The oxygen concentrations are expressed as $\mathrm{mol} \mathrm{m}^{-3}$. According to the tables of Riley \& Skirrow (1975), at $20^{\circ} \mathrm{C}$ the concentration of oxygen in water equilibrated with air containing $29.05 \% \mathrm{O}_{2}$ is 0.00635 by volume, equivalent to $0.264 \mathrm{~mol} \mathrm{~m}^{-3}$. The concentration in equilibrium with an atmosphere of $100 \%$ oxygen would therefore be $0.909 \mathrm{~mol} \mathrm{~m}^{-3}$. The oxygen concentration gradient from the surface of the gas vesicle suspension to the bottom of the agar layer would therefore have ranged between these two values.

\section{Results}

\section{Oxygen diffusion gradients}

Three profiles of oxygen concentration were measured: the mean of the three measurements is shown in Fig. 1. In each profile a change in the slope of the oxygen concentration gradient was observed about $1 \mathrm{~mm}$ below the surface of the gas vesicle suspension, a depth which corresponds with the upper surface of the agar layer. Below this position the concentration gradients were linear (in each case the correlation coefficient $r>0.99$ ). The mean value for the gradients of these concentration profiles in agar, $\mathrm{d} C / \mathrm{d} x$, was $0.252 \mathrm{~mol} \mathrm{~m}^{-3} \mathrm{~mm}^{-1}$. Above this position the concentration gradients in the gas vesicle suspension were also linear, to within $0.2 \mathrm{~mm}$ of the suspension-air interface; the mean value of the gradients, $\mathrm{d} C_{1} / \mathrm{d} x$, was $0.311 \mathrm{~mol} \mathrm{~m}^{-3} \mathrm{~mm}^{-1}$.

The ratio of the gradients in the gas vesicle suspension and in agar was therefore $0.311 / 0 \cdot 252$ or $1 \cdot 23$. It follows from equation (1) that the ratio of the diffusivities of oxygen gas through the gas vesicle suspension $\left(D_{\mathrm{s}}\right)$ and through agar, or water $\left(D_{\mathrm{w}}\right)$, is the reciprocal of this ratio, i.e. $D_{\mathrm{s}}=0.812 D_{\mathrm{w}}$.

\section{The volume fraction of gas vesicles in the suspension}

The volume of gas space in the gas vesicle suspension was determined from measurements in the compression tube. A sample of volume $30 \mu \mathrm{l}$ was diluted to $10 \mathrm{ml}$; a 
compression tube of volume $1571 \mu \mathrm{l}$ was filled with the diluted suspension and exposed to pressure. The mean linear contraction in the attached capillary tube was $45.97 \pm 0.18 \mathrm{~mm}$ (four measurements), equivalent to a volumetric contraction of $1.457 \mathrm{~mm}^{3}$. This represents a gas space of $9.27 \mu \mathrm{l}$ in the original $30 \mu \mathrm{l}$ of suspension, or 0.309 of the suspension volume.

Measurements made by electron microscopy on the gas vesicles of the same strain of Microcystis show that the ratio of total gas vesicle volume to gas volume is $1 \cdot 201 / 1.063$ (see Table 1 of Walsby \& Bleything, 1988). From this it is calculated that the gas vesicles represented $\phi_{\mathrm{v}}=0.349$ of the suspension volume. The remainder, $\phi_{\mathrm{w}}=0.651$, was water.

\section{Comparison with diffusion gradients through suspensions of glass beads}

In a similar experimental procedure Revsbech (1989) showed that the ratio $(R)$ of the diffusion gradients of oxygen gas through a suspension of densely packed glass beads and through agar was $R=3.68$ when the volume fraction of water in the suspension was $\phi_{w}=0.378$. The expected ratio with a glass bead suspension containing water of volume fraction $\phi_{\mathrm{w}}=0.651$, the same as for the gas vesicle suspension, would have been $R=3.68 \times 0.378 / 0.651=2.14$ (i.e. making the assumption that diffusivity varies with the volume fraction of water). Above an agar layer with a gradient of $0.252 \mathrm{~mol}$ $\mathrm{m}^{-3} \mathrm{~mm}^{-1}$ the expected gradient in such a suspension of glass beads (through which no gas diffusion occurs) would have been $0.54 \mathrm{~mol} \mathrm{~m}^{-3} \mathrm{~mm}^{-1}$. This is shown in Fig. 1 for comparison with the gradient in the gas vesicle suspension, which is seen to be closer to the extrapolated gradient in agar. It is concluded that gas diffusion through the gas vesicles produces a marked difference in diffusivity through the layer.

\section{Discussion}

\section{The diffusivity of oxygen through the gas vesicle}

The first conclusion that can be drawn from the comparison between the diffusion gradients in the gas vesicle suspension and in water (agar) is that, since the gradient is steeper in the suspension, the gas vesicles must be less permeable to oxygen than the water they displace.

It is shown in Appendix A that from measurements of the fraction of the suspension volume occupied by water and by gas vesicles it is possible to determine $D_{v}$, the diffusivity through an imaginary substance that, formed into particles occupying the same volume fraction as the gas vesicles, would give a suspension in which the diffusivity would be the same as in the gas vesicle suspension, $D_{\mathrm{s}}=0.812 D_{\mathrm{w}}$. From diffusion theory (see equations $\mathrm{A} 2$ and $\mathrm{A} 3$ ), the value of $D_{\mathrm{v}}$ lies between the limits of $0.518 D_{\mathrm{w}}$ and $0.539 D_{\mathrm{w}}$. In the following calculations the mean value of $0.528 D_{\mathrm{w}}$ is adopted, which has an error of not more than $\pm 0.011 D_{\mathrm{w}}$, about $2 \%$. At the temperature of the measurement, $20^{\circ} \mathrm{C}, D_{\mathrm{w}}$ is $2.0 \times 10^{-9} \mathrm{~m}^{2} \mathrm{~s}^{-1}$; it follows that $D_{\mathrm{v}}=1.06 \times 10^{-9} \mathrm{~m}^{2} \mathrm{~s}^{-1}$ (or $1.06 \times 10^{3} \mu \mathrm{m}^{2} \mathrm{~s}^{-1}$ ). It should be noted here that while the overall diffusivity in the suspension of particles would be the same as the diffusivity in the gas vesicle suspension, the nature of the diffusion through the solid particles and the hollow gas vesicles would be different. In the solid particle the diffusion gradient $(\mathrm{d} C / \mathrm{d} x)$ would be uniform, whereas in the gas vesicle the gradient would be much steeper across the walls than across the intervening gas space. For this reason the term $D_{\mathrm{v}}$ is a theoretical abstraction; we refer to it as the notional diffusivity in the gas vesicle.

The same experimental procedure could be used to determine the diffusivity of oxygen through other types of particles, from measurements of the diffusion gradients through concentrated suspensions. If the particles were homogeneous the value $D_{\mathrm{v}}$ would give the actual diffusivity in the substance of the particle. It would be of interest to determine the diffusivity through whole cells and through carboxysomes, which, it has been speculated, may have a differential diffusivity to oxygen and carbon dioxide.

\section{The gas permeability coefficient of the gas vesicle wall}

In Appendix B it is demonstrated that, neglecting end effects, a long solid cylinder, made of a substance in which the diffusivity is $D_{v}$, has the same effect on diffusion through a suspension as a hollow (gas-filled) cylinder when

$$
D_{\mathrm{v}}=\kappa a
$$

where $\kappa$ is the permeability coefficient of the wall of the hollow cylinder, and $a$ is the cylinder radius. This conclusion holds for any orientation at which the two cylinders are considered, and it will therefore be valid for suspensions of randomly oriented cylinders. We use this result to obtain an estimate of the permeability coefficient of the wall of the Microcystis gas vesicle, which, although not infinitely long, has a high aspect ratio, so that diffusion through the side walls will dominate over that through the end walls. The cylinder radius of the Microcystis gas vesicle is $a=32.6 \times 10^{-9} \mathrm{~m}$ (Walsby $\&$ Bleything, 1988) and hence the approximate value of the 
gas permeability coefficient of the gas vesicle wall given by equation (2) is $\kappa=0.032 \mathrm{~m} \mathrm{~s}^{-1}$ (or $32 \times 10^{3} \mu \mathrm{m} \mathrm{s}^{-1}$ ). This estimate of the permeability coefficient exceeds the minimum limit, $332 \mu \mathrm{m} \mathrm{s}^{-1}$, indicated by the pressurerise experiments of Walsby (1984) by nearly two orders of magnitude.

The rate constant, $\alpha$, for the gas vesicle to fill with gas by diffusion is defined by the expression

$$
\alpha=\kappa A_{\mathrm{o}} / V_{\mathrm{i}}
$$

where $A_{0}$ is the outer surface area and $V_{1}$ the internal (gas) volume of the gas vesicle (Walsby, 1984). For the Microcystis gas vesicle in which $A_{\mathrm{o}}=0.792 \times 10^{5} \mathrm{~nm}^{2}$ and $V_{\mathrm{i}}=1.063 \times 10^{6} \mathrm{~nm}^{3}$ (calculated from the data in Table 1 of Walsby \& Bleything, 1988), $\alpha=2.42 \times 10^{6} \mathrm{~s}^{-1}$. The 1/e folding time, $t_{\mathrm{e}}$, for the gas vesicle to fill (to a pressure $p / e)$ by diffusion is therefore given by $1 / \alpha=0.41 \times 10^{-6} \mathrm{~s}$.

Gas vesicles of different cyanobacteria have similar molecular constituents (Walsby \& Hayes, 1989) and the value of $\kappa$ in the different species should therefore be similar. The rate constant, $\alpha$, is size-dependent, however (equation 3). For the larger Anabaena gas vesicle, in which $A_{\mathrm{o}}=1.20 \times 10^{5} \mathrm{~nm}^{2}$ and $V_{\mathrm{i}}=2.14 \times 10^{6} \mathrm{~nm}^{3}$ (from Walsby \& Bleything, 1988), $\alpha=1.82 \times 10^{6} \mathrm{~s}^{-1}$ and $t_{\mathrm{e}}=0.55 \mu \mathrm{s}$. The essential conclusion for all gas vesicles is that the equilibration times for gases diffusing in and out are exceedingly short.

\section{Diffusion across layers of gas vesicles}

In the cells of cyanobacteria, gas vesicles are usually stacked together in layers, such that the long axes of the individual gas vesicles lie parallel to one another. The effective diffusivity though such a layer in a direction parallel to the long axis of the gas vesicles will be higher than that at right angles, because a greater length of gas space will be crossed between the two walls. We will first determine the diffusivity through a layer in which it is assumed that the interstitial spaces between the gas vesicles are filled with gas, and we will then consider a more realistic model of the gas vacuole where the interstitial spaces are filled with water.

When the diffusion gradient is parallel to the long axis the width of the layer between two gas vesicle walls will be approximately equal to the length of the gas vesicle, $L$; since the diffusion resistance of the gas space is negligible, the longitudinal diffusivity will be

$$
D_{1}=\kappa L / 2
$$

Then, since $\kappa=D_{\mathrm{v}} / a$ and $D_{\mathrm{v}}=0.528 D_{\mathrm{w}}$, it follows that

$$
D_{1}=0.264 D_{\mathrm{w}} L / a
$$

For the Microcystis gas vesicle, in which $L=430 \mathrm{~nm}$ and $a=32.6 \mathrm{~nm}, D_{1}=3.5 D_{\mathrm{w}}$; i.e. the gas will diffuse 3.5 times more rapidly through such a hypothetical gas vesicle layer in this direction than through an equivalent length of water. In this orientation the gas vesicle may therefore act as a diffusion channel.

When the diffusion gradient is perpendicular to the long axis the average distance between adjacent layers of cylinders, hexagonally stacked, is $h=a \sqrt{ } 3=1.73 a$. Again, the diffusing gas must pass two walls and the effective transverse diffusivity through the gas vesicle layer would be

$$
D_{\mathrm{t}}=\left(\kappa a_{\vee} 3\right) / 2
$$

Then, substituting as in equation (5),

$$
D_{\mathrm{t}}=0.457 D_{\mathrm{w}}
$$

i.e. the gas will diffuse about twice as quickly through water as through the gas vesicle in this orientation.

\section{Diffusion across gas vacuoles}

In gas vacuoles the gas vesicles are hexagonally stacked, with the interstitial spaces filled with cytoplasmic fluid, which can be assumed to be water. In such an arrangement, flat-ended cylinders occupy a proportion $\phi_{9}=\pi / 2 \sqrt{ } 3$, or 0.907 , of the area in transverse section, and hence also of the volume. The overall diffusivity parallel to the longitudinal axis of the gas vesicles in such a stack, in which the gas may diffuse either via the gas vesicles or by the contiguous channels of interstitial water (of volume $\phi_{\mathrm{i}}=1-\phi_{\mathrm{g}}$ ) between them, is given by

$$
D_{\mathrm{L}}=\phi_{\mathrm{i}} D_{\mathrm{w}}+\phi_{\mathrm{g}} D_{\mathrm{l}}
$$

Then, when $\phi_{9}=0.907, \phi_{\mathrm{i}}=0.093$, and $D_{1}=3.5 D_{\mathrm{w}}, D_{\mathrm{L}}$ is calculated to be $3 \cdot 3 D_{\text {w }}$.

When the diffusion gradient is at right-angles to the longitudinal axis of gas vesicles in the stack, the diffusing gas must pass through each gas vesicle layer as there is no alternative free channel between the gas vesicles. The overall diffusivity is then given approximately by the expression

$$
D_{\mathrm{T}}=D_{\mathrm{t}} D_{\mathrm{w}} /\left(\phi_{\mathrm{g}} D_{\mathrm{w}}+\phi_{\mathrm{i}} D_{\mathrm{t}}\right)
$$

and substituting the values of $D_{\mathrm{t}}, D_{\mathrm{w}}, \phi_{9}$ and $\phi_{\mathrm{i}}$ used previously, we obtain $D_{\mathrm{T}}=0.48 D_{\mathrm{w}}$. This is slightly higher than the diffusivity transversely across the single gas vesicle, because of the faster diffusion through the small volume of interstitial water.

\section{Diffusivity through gas vesicles of other cyanobacteria}

The gas vesicles of other cyanobacteria have a similar shape but different dimensions (Walsby \& Bleything, 1988). The narrowest gas vesicles, which occur in 
Trichodesmium thiebautii, have a cylinder radius $a=22.5 \mathrm{~nm}$ (Gantt et al., 1984). Were they to have a wall of the same permeability coefficient of $\kappa=0.0324 \mathrm{~m} \mathrm{~s}^{-1}$, we should expect a notional diffusivity of only $D_{\mathrm{v}}=650 \mu \mathrm{m}^{2} \mathrm{~s}^{-1}$. With the widest cyanobacterial gas vesicles, in Dactylococcopsis salina, in which $a=54.7 \mathrm{~nm}$, we would expect a notional diffusivity of $1800 \mu \mathrm{m}^{2} \mathrm{~s}^{-1}$, which is still less than the diffusivity in water. It is possible that some of the gas vesicles in halobacteria may be so wide (see Walsby, 1972) that the notional diffusivity is higher than the diffusivity in water, but they have a rather different, spindle-shaped morphology; direct investigation of their permeability would be needed.

\section{Speculations on the number of pores and their size}

A gas molecule permeating a membrane must find its way through spaces between or within the molecules that form the structure of the membrane; the spaces may change size due to molecular vibration, and so admit molecules larger than the time-averaged size of these pores (Glazer, 1976; Scouloudi, 1969). Walsby (1984) discussed the number of pores of a given size that would be needed to explain a given permeability coefficient of a gas vesicle, based on molecular collision theory.

The starting point was that there exists in the gas vesicle at least one pore, assumed to be a circle of radius $f$. The gas molecule, of collision radius $r_{\sigma}$, would pass through the pore if its centre passed through an effective window of area $w$, which can be determined by averaging over all trajectories (see Appendix C). The collision frequency of a single molecule with the inside of the gas vesicle $A_{\mathrm{i}}$ was shown to be

$$
\mathrm{d} N_{\mathrm{s}} / \mathrm{d} t=A_{\mathrm{i}}\langle v\rangle / 4 V_{\mathrm{i}}
$$

where $A_{\mathrm{i}}$ is the inside area of the wall, $V_{\mathrm{i}}$ the inside volume of the vesicle, and $\langle v\rangle=(8 R T / \pi M)^{1 / 2}$, the average velocity of the gas molecule, which for oxygen at $20^{\circ} \mathrm{C}$ is $440 \mathrm{~m} \mathrm{~s}^{-1}$ (see equation 13 of Walsby, 1984). It was also shown by probability theory that in the time $\tau_{\mathrm{e}}$ it takes for $1 / \mathrm{e}$ of the gas molecules to escape from a single pore, each molecule makes on average $A_{\mathrm{i}} / w$ collisions with the wall, so that the collision frequency is also given by

$$
\mathrm{d} N_{\mathrm{s}} / \mathrm{d} t=A_{\mathrm{i}} / w \tau_{\mathrm{e}}
$$

Combining (11) and (12), the folding time for a molecule to escape by diffusion from a single pore is

$$
\tau_{\mathrm{e}}=4 V_{\mathrm{i}} / w\langle v\rangle
$$

If the number of such pores in the gas vesicle is $N_{\mathrm{p}}$, then the folding time for the gas molecules to escape is

$$
t_{\mathrm{e}}=4 V_{\mathrm{i}} / N_{\mathrm{p}} w\langle v\rangle
$$

The reciprocal of $t_{\mathrm{e}}$ gives the rate coefficient $\alpha$, which is therefore $N_{\mathrm{p}} w\langle v\rangle / 4 V_{\mathrm{i}}$. Now, since $\alpha=\kappa A_{\mathrm{o}} / V_{\mathrm{i}}$ (equation 3 ), the permeability coefficient $\kappa$ depends on the effective area of the pores and the number of pores in the gas vesicle wall:

$$
\kappa=N_{\mathrm{p}} w\langle v\rangle / 4 A_{\mathrm{o}}
$$

There are two unknowns in this equation, $N_{\mathrm{p}}$ and $w$. We can speculate on the number of pores, however, and then calculate for a given value of $N_{\mathrm{p}}$ the size of those pores.

It would be expected that in a crystalline structure like the gas vesicle each pore would be associated with a periodic structure, e.g. there might be one pore per rib or one pore associated with each GvpA molecule. The argument is now transferred to the Anabaena gas vesicle, in which more is known of the molecular structure. It has an outer wall area of $A_{\mathrm{o}}=1.20 \times 10^{5} \mathrm{~nm}^{2}$ and a wall of volume $0.211 \times 10^{6} \mathrm{~nm}^{3}$ (Walsby \& Bleything, 1988) which, composed of protein with a density of $1320 \mathrm{~kg}$ $\mathrm{m}^{-3}$ (Blaurock \& Walsby, 1976), gives a wall mass of $M_{\mathrm{w}}=2.79 \times 10^{-16} \mathrm{~g}$. Of this, 0.917 is accounted for by GvpA (Walsby \& Hayes, 1989), which has an $M_{\mathrm{r}}$ of 7397 (Hayes et al., 1988). The number of GvpA molecules is therefore $N_{\mathrm{a}}=(0.917 / 7397) N_{\mathrm{A}} M_{\mathrm{w}}=20857\left(N_{\mathrm{A}}\right.$ is the Avogadro number). Substituting this value of $N_{\mathrm{a}}$ for $N_{\mathrm{p}}$ in equation (14) gives the area of the effective window of each pore as $w=0.0017 \mathrm{~nm}^{2}$; the radius of such a pore is calculated to be between 0.20 and $0.22 \mathrm{~nm}$ (Appendix $\mathrm{C}$ ). It is noted that a pore of diameter $0.44 \mathrm{~nm}$ would be accommodated adjacent to the ends of the $\beta$-chains in a model of the GvpA molecule proposed by E. D. T. Atkins \& A. E. Walsby (see Fig. 6 in Walsby \& Hayes, 1989). It is also noted, though, that the diameter of such a pore is less than the $0.63 \mathrm{~nm}$ collision diameter of the perfluorocyclobutane molecule, which has been shown to permeate the gas vesicle (Walsby, 1982b). This does not eliminate the possibility that the pore has a timeaveraged diameter that is smaller than $0.63 \mathrm{~nm}$, however, as it may be open wide enough to admit the molecule for a small proportion of the time (Walsby, 1984).

The number of ribs in the gas vesicle is given by $N_{\mathrm{r}}=L / R$, where $L$ is the overall length, $494 \mathrm{~nm}$, and $R$ is the rib periodicity, $4.6 \mathrm{~nm}$; hence $N_{\mathrm{r}}=107$. Substituting this value of $N_{\mathrm{r}}$ for $N_{\mathrm{p}}$ in equation (14) gives the area of the effective window of each pore as $w^{\prime}=0.33 \mathrm{~nm}^{2}$, and the radius of the pore as between 0.50 and $0.59 \mathrm{~nm}$ (Appendix C). The diameter of such a pore, $1.18 \mathrm{~nm}$, would be similar in size to the $1.15 \mathrm{~nm}$ crystallographic repeat of the GvpA molecule along the ribs (Blaurock \& Walsby, 1976).

With crystallography of higher resolution it may be possible to determine the location and number of pores in the gas vesicle wall that can account for the observed permeability coefficient. 
We thank Mrs Annette Richer for culturing the cyanobacteria. This work was supported by a grant from the Natural Environment Research Council.

\section{Appendix A}

\section{Calculation of the notional diffusivity in randomly oriented gas vesicles}

This appendix discusses the relation between the measured diffusivity of the suspension and the notional diffusivity of gas through the gas vesicles, discussed under the heading 'The diffusivity of oxygen through the gas vesicle'. The theory of the diffusivity in a mixture of two substances goes back to Maxwell (1873). He was studying electricity, but the results are immediately applicable here since the flow of electricity in a conductor is governed by the same equations as the diffusion of gas through a suspension. Maxwell showed that if spheres in which the diffusivity is $D_{1}$ are suspended in a medium in which the diffusivity is $D_{2}$, then the overall diffusivity $D_{\mathrm{s}}$ in the suspension is

$$
D_{\mathrm{s}}=D_{2}\left[D_{1}\left(1+2 \phi_{1}\right)+2 D_{2} \phi_{2}\right] /\left[D_{2}\left(2+\phi_{1}\right)+D_{1} \phi_{2}\right]
$$

where $\phi_{1}$ is the fraction of the total volume occupied by the spheres and $\phi_{2}=1-\phi_{1}$ is the volume fraction for the second medium. This formula was derived on the assumption that the spheres are far enough apart to act independently. But experiments by Turner (1976) show that it applies however closely packed the spheres are, provided that $D_{1} / D_{2}$ is not too large or small.

Gas vesicles are not spheres, of course, so the Maxwell formula does not apply. However, results which are independent of the shape or orientation of the gas vesicles follow from Hashin \& Shtrikman (1962); they worked on the theory of magnetic permeability, which again is mathematically identical to diffusion theory. They considered a medium which appears homogeneous and isotropic when viewed on a macroscopic scale, while being a mixture of two substances on the microscopic scale. In this case they proved that the diffusivity in the mixture will always lie between two extreme values, $D_{\mathrm{s}}^{\prime}$ and $D_{\mathrm{s}}^{\prime \prime}$, defined as follows:

$$
\begin{aligned}
& D_{\mathrm{s}}^{\prime}=D_{2}\left[D_{1}\left(1+2 \phi_{1}\right)+2 D_{2} \phi_{2}\right] /\left[D_{2}\left(2+\phi_{1}\right)+D_{1} \phi_{2}\right] \\
& D_{\mathrm{s}}^{\prime \prime}=D_{1}\left[D_{2}\left(1+2 \phi_{2}\right)+2 D_{1} \phi_{1}\right] /\left[D_{1}\left(2+\phi_{2}\right)+D_{2} \phi_{1}\right]
\end{aligned}
$$

Comparing (A2) and (A1) shows that $D_{\mathrm{s}}^{\prime}$ corresponds to medium 1 being concentrated into spheres embedded in medium $2 ; D_{\mathrm{s}}^{\prime \prime}$ corresponds to medium 2 concentrated into spheres embedded in medium 1.
For the moment we model the gas vesicles as homogeneous solid structures, formed of an imaginary substance in which the diffusivity is $D_{v}$, that give a suspension with the same diffusive properties as the gas vesicle suspension. (In Appendix B we extend the theory to a realistic model of a vesicle.) We determine the first extreme value of $D_{v}$ from (A2) by taking $D_{1}=D_{v}$, $D_{2}=D_{\mathrm{w}}$ (the diffusivity in water), $D_{\mathrm{s}}=0.812 D_{\mathrm{w}}$, $\phi_{1}=\phi_{\mathrm{v}}=0.349$, and $\phi_{2}=\phi_{\mathrm{w}}=0.651$ (the values from the experiment). Solving for $D_{1}$ gives the lower limit of $D_{\mathrm{v}}=0.518 D_{\mathrm{w}}$. In the same way we determine the second extreme value of $D_{\mathrm{v}}$ from (A3) by substituting the same values of $D_{2}, \phi_{1}$ and $\phi_{2}$, and $D_{\mathrm{s}}=0.812 D_{\mathrm{w}}$; solving for $D_{1}$ then gives the upper limit of $D_{\mathrm{v}}=0.539 D_{\mathrm{w}}$. The true value of $D_{v}$ must lie between these two extremes; the value adopted is the value midway between them, $D_{\mathrm{v}}=0.526 D_{\mathrm{w}}$.

This result is independent of the shape of these solid structures and is therefore also independent of their orientation. The only assumption made is that the suspension was homogeneous and isotropic (i.e. randomly oriented).

\section{Appendix B}

The relationship between diffusivity and the permeability coefficient

This Appendix compares a solid cylinder of uniform diffusivity $D_{v}$ with a hollow cylinder that has a wall permeability $\kappa$. The two cylinders will be shown to form suspensions with the same overall diffusivity if $\kappa a=D_{v}$, where $a$ is the radius of the cylinder. The cylinder will be assumed to be so long that end-effects may be neglected; this is a reasonable approximation for the gas vesicles under consideration here.

Consider a uniform medium, say a tank of water, containing oxygen with a constant concentration gradient, producing a constant unidirectional flux. If a cylinder is placed in the middle of the tank, the oxygen flow will be disturbed, but eventually will settle down to a steady flux. If the cylinder is much smaller than the tank, then near the edge of the tank the flux will be uniform, essentially unaffected by the cylinder. In the neighbourhood of the cylinder the flow will curve towards or away from it, depending on its diffusivity relative to water. We shall calculate this distortion of the flow for the two types of cylinder.

\section{(i) Solid cylinder}

Consider an infinitely long cylinder of radius $a$ and diffusivity $D_{\mathrm{v}}$ embedded in a uniform medium of 
diffusivity $D_{\mathrm{w}}$ representing the water in which the vesicle is immersed. We shall first suppose that the concentration gradient in the medium is perpendicular to the axis of the cylinder.

In order to calculate the flow, a coordinate system is required. A general point $\mathrm{P}$ has polar coordinates $r, \theta$, where $r$ is the distance of $\mathrm{P}$ from the axis (more precisely, from the point $O$ on the axis which is closest to $P$ ), and $\theta$ is the angle between OP and the direction of the concentration gradient. Thus $r=a$ represents the surface of the cylinder, and $r \cos \theta$ is distance measured along the concentration gradient.

Let $G$ be the (constant) concentration gradient far away from the cylinder. Then the concentration $c$ far from the cylinder is given by $c=\bar{C}+G r \cos \theta$, where the constant $\bar{C}$ is the average concentration, and the second term gives the gradient. Because of the cylinder, the concentration gradient is not uniform everywhere; it is determined by the differential equations of diffusion. In this case we have Laplace's equation $\nabla^{2} c=0$ inside and outside the cylinder. Also we require that the concentration $c$ and the flux $D \partial c / \partial r$ match across the surface, where $D$ is the appropriate diffusivity. Thus $c(r=a+)=$ $c(r=a-)$ and $D_{\mathrm{w}} \partial c / \partial r(r=a+)=D_{\mathrm{v}} \partial c / \partial r(r=a-)$, where the notation $r=a+$ refers to values just outside the surface at $r=a$, and $r=a-$ means just inside. For background on partial differential equations see, for example, Boas (1983); for details of the diffusion equation see Crank (1975).

The solution satisfying the above conditions is

$$
c=\bar{C}+G\left\{r+[(1-Q) /(1+Q)] a^{2} / r\right\} \cos \theta
$$

outside the cylinder, and

$$
c=\bar{C}+G\{2 /(1+Q)\} r \cos \theta
$$

inside the cylinder, where

$$
Q=D_{\mathrm{v}} / D_{\mathrm{w}}
$$

is the ratio of the diffusivities inside and outside the cylinder. It is easy to verify that this solution satisfies all the required conditions.

\section{(ii) Hollow cylinder}

Now consider a hollow cylinder, with wall permeability $\kappa$, which is similarly oriented so that the concentration gradient is perpendicular to the axis of the cylinder. Again the concentration far from the cylinder is
$c=\bar{C}+G r \cos \theta$. Inside the hollow cylinder the concentration is constant, $c=\bar{C}$. The concentration outside is again determined by Laplace's equation with appropriate boundary conditions.

The concentrations just inside and outside the wall need not match; instead of the matching conditions in $(i)$ above, we require that the flux through the wall equal the flux just outside: $\kappa[c(r=a+)-c(r=a-)]=$ $D_{\mathrm{w}} \partial c / \partial r(r=a+)$. Applying this condition gives the solution

$$
c=\bar{C}+G\left\{r+[(1-S) /(1+S)] a^{2} / r\right\} \cos \theta
$$

outside the cylinder, where

$$
S=\kappa a / D_{\mathrm{w}}
$$

\section{(iii) Conclusion}

The formulae (B1) and (B4) will be equivalent when $Q=S$. In view of (B3) and (B5) this means

$$
\kappa a=D_{\mathrm{v}}
$$

This is the condition required for a solid cylinder of radius $a$, in which the diffusivity is $D_{v}$, to have the same effect as a hollow cylinder with wall permeability $\kappa$, in circumstances where gas is diffusing steadily through the cylinder oriented perpendicular to the diffusion gradient.

At all other orientations of the cylinders the flow may be resolved into components parallel and perpendicular to the axis of the cylinder. Diffusion parallel to the axis, in the region outside the cylinder, is unaffected by the presence of the cylinder. It is therefore concluded that equation (B6) is true for any orientation in which the solid and hollow cylinder are compared.

\section{Appendix C}

\section{The relation between diameter and effective area of a pore}

In order to calculate the probability of a spherical gas molecule (of radius $r_{\sigma}$ ) escaping through a circular pore (of radius $f$ ) through the wall of a gas vesicle, Walsby (1984) calculated the effective area $(w)$ of the pore through which the centre of the molecule must pass.

In a geometrical analysis, in which it is assumed that the sphere escapes as long as no contact is made with the rim of the pore, the effective area, averaged for trajectories from all angles and directions is given by equation $(\mathrm{C} 1)$.

$$
w=\frac{4}{\pi} \int_{0}^{f-r} \int_{\theta_{\mathrm{m}}}^{\pi / 2}\left\{\left[\left(r_{\sigma}^{2}-y^{2}\right)^{1 / 2}\left(b \sin ^{2} \theta-y \cos ^{2} \theta\right) / y \sin \theta\right]+\left[\left(f-r_{\sigma}\right)^{2}-b^{2}\right]^{1 / 2}\right\} \cos \theta \mathrm{d} \theta \mathrm{d} b
$$


This is a modification of equation (A21) of Walsby (1984) with two typographical corrections: $(a)$ the sign in the term $\left(f-r_{\sigma}\right)$ is corrected: $(b)$ the position of $\mathrm{d} \theta$ is moved to include the last term in the integration over different angles. (The value of $w=0.0301 \mathrm{~nm}^{2}$ was correctly calculated, free of these errors.) A third modification is the insertion of the term $\cos \theta$ at the end of the integral to give the correct weighting for the different frequencies of molecules arriving at the window from different angles.

Equation $(\mathrm{C} 1)$ can be solved by numerical methods to determine the value of $f$ for given values of $r_{\sigma}$ and $w$. The oxygen gas molecule has a collision diameter of $\sigma=0.346 \mathrm{~nm}$ (Breck, 1974) and hence a radius of $r_{\sigma}=0.173 \mathrm{~nm}$. In the Discussion we considered the case where there is one pore per GvpA molecule, when $w=0.0017 \mathrm{~nm}^{2}$; this value is given by pore of radius $f=0.22 \mathrm{~nm}$. We also considered the case where there is one pore per rib, when $w=0.33 \mathrm{~nm}^{2}$; this value is given by a pore of radius $f=0.59 \mathrm{~nm}$.

By statistical mechanics (Jeans, 1940) it can be argued that the molecule will enter if its centre passes through a circle of radius $\left(f-r_{\sigma}\right)$; the area of the effective window is then

$$
w=\pi\left(f-r_{\sigma}\right)^{2}
$$

and therefore

$$
f=r_{\sigma}+\vee(w / \pi)
$$

Then, when $w=0.0017 \mathrm{~nm}^{2} ; f=0.20 \mathrm{~nm}$, and when $w=0.33 \mathrm{~nm}^{2}, f=0.50 \mathrm{~nm}$. These values of $f$ are slightly lower because in this argument some additional trajectories involving collisions with the rim of the pore also permit escape of the molecule.

\section{References}

Blaurock, A. E. \& Walsby, A. E. (1976). Crystalline structure of the gas vesicle wall from Anabaena flos-aquae. Journal of Molecular Biology 105, 183-199.

BoAs, M. L. (1983). Mathematical Methods in the Physical Sciences, 2nd edn. New York: Wiley.

BRECK, D. W. (1974). Zeolite Molecular Sieves. New York: Wiley.

Carpenter, E. J. \& Price, C. C. (1976). Marine Oscillatoria (Trichodesmium): explanation for aerobic nitrogen fixation without heterocysts. Science 191, 1278-1280.

CRANK, J. (1975). The Mathematics of Diffusion, 2nd edn. Oxford: Oxford University Press.
GantT, E., OKHI, K. \& FujITA. Y. (1984). Trichodesmium thiebautii; structure of a nitrogen-fixing marine blue-green alga (Cyanophyta). Protoplasma 119, 188-196.

Glazer, A. N. (1976). Chemical modification of proteins. In The Proteins, vol. 2, pp. 1-103. Edited by H. Newrath \& R. L. Hill. New York: Academic Press.

Hashin, Z. \& Shtrikman, S. (1962). A variational approach to the theory of the effective magnetic permeability of multiphasic materials. Journal of Applied Physics 33, 3125-3131.

HAYES, P. K. (1988). Gas vesicles: chemical and physical properties. Methods in Enzymology 167, 213-222.

hayes, P. K., Lazarus, C. M., Bees, A., Walker, J. E. \& Walsby, A. E. (1988). The protein encoded by $g v p C$ is a minor component of gas vesicles isolated from the cyanobacteria Anabaena flos-aquae and Microcystis sp. Molecular Microbiology 2, 545-552.

JEANS, J. (1940). An Introduction to the Kinetic Theory of Gases. Cambridge: Cambridge University Press.

MaXwell, J. C. (1873). A Treatise on Electricity and Magnetism. Oxford: Oxford University Press.

RevsBech, N. P. (1989). Diffusion characteristics of microbial communities determined by use of oxygen microsensors. Journal of Microbial Methods 9, 111-122.

REVSBECH, N. P. \& W ARD, D. M. (1983). Oxygen microelectrode that is insensitive to medium chemical composition: use in an acid microbial mat dominated by Cyanidium caldarium. Applied and Environmental Microbiology 45, 755-759.

Riley, J. P. \& SKIRrow, G. (1975). Chemical Oceanography vol. 1, p. 561. London: Academic Press.

Scouloudi, H. (1969). X-ray crystallographic studies of seal myoglobin : the model at $6 \AA$ and $5 \AA$ resolution. Journal of Molecular Biology 40. 353-377.

TAYLOR, B. F., LeE, C. C. \& BunT, J. S. (1973). Nitrogen-fixation associated with the marine blue-green alga, Trichodesmium, as measured by the acetylene-reduction technique. Archiv für Mikrobiologie 88, 205-212.

TURNER, J. C. R. (1976). Two-phase conductivity. The electrical conductance of liquid-fluidised beds of spheres. Chemical Engineering Science 31, 487-492.

WALSBY, A. E. (1969). The permeability of blue-green algal gas-vacuole membranes to gas. Proceedings of the Royal Society of London B173, 235-255.

WALSBY, A. E. (1971). The pressure relationships of gas vacuoles. Proceedings of the Royal Society of London B178, 301-326.

WALSBY, A. E. (1972). Structure and function of gas vacuoles. Bacteriological Reviews 36, 1-32.

W ALSBY, A. E. (1974). The isolation of gas vesicles from blue-green algae. Methods in Enzymology 31, 678-686.

W ALSBY, A. E. $(1982 a)$. The elastic compressibility of gas vesicles. Proceedings of the Royal Society of London B216, 355-368.

W ALSBY, A. E. (1982b). Permeability of gas vesicles to perfluorocyclobutane. Journal of General Microbiology 128, 1679-1684.

W ALSBY, A. E. (1984). Lower limit of the gas permeability coefficient of gas vesicles. Proceedings of the Royal Society of London B223, 177. 196.

WaLSBY, A. E. (1985). The permeability of heterocysts to the gases nitrogen and oxygen. Proceedings of the Royal Society of London B226, 345-366.

W ALSBY, A. E. (1991). The mechanical properties of the Microcystis gas vesicle. Journal of General Microbiology 137, 2401-2408.

WalsBy, A. E. \& BLEything, A. (1988). The dimensions of cyanobacterial gas vesicles in relation to their efficiency in providing buoyancy and withstanding pressure. Journal of General Microbiology 134. 2635-2645.

WalsBy, A. E. \& HAyes, P. K. (1989). Gas vesicle proteins. Biochemical Journal 264, 313-322. 